\title{
Data-driven analysis methods for the measurement of reconstructed jets in heavy ion collisions at RHIC and LHC
}

\author{
G. O. V. de Barros ${ }^{\mathrm{a}}$, Bo Fenton-Olsen ${ }^{\mathrm{b}}$, Peter Jacobs ${ }^{\mathrm{b}, \mathrm{c}}$, Mateusz Płoskoń ${ }^{\mathrm{b}}$ \\ ${ }^{a}$ Universidade de São Paulo, Instituto de Física \\ Rua do Matão Travessa R, 187, 05508-090, São Paulo, Brazil \\ ${ }^{b}$ Lawrence Berkeley National Laboratory \\ 1 Cyclotron Road, Berkeley, CA 94720, USA \\ ${ }^{c} C E R N$
}

CH-1211 Geneva 23, Switzerland

\begin{abstract}
We present data-driven methods for the full reconstruction of jets in heavy ion collisions, for inclusive and coincidence jet measurements at both RHIC and LHC. The complex structure of heavy ion events generates a large background of combinatorial jets, and smears the measured energy of the true hard jet signal. Techniques to correct for these background effects can induce biases in the reported jet distributions, which must be well controlled for accurate measurement of jet quenching. Using model studies, we evaluate the proposed methods for measuring jet distributions accurately while minimizing the fragmentation bias of the measured population.
\end{abstract}

Keywords: Jet Reconstruction, Heavy Ion Collisions, Iterative Bayesian Unfolding

\section{Introduction}

The interaction of QCD jets with the Quark-Gluon Plasma (QGP) generated in high energy nuclear collisions (“jet quenching") provides unique and penetrating probes of the QGP. Jet quenching has to date been measured primarily via the suppression of high $p_{\mathrm{T}}$ hadrons and their correlations [1]. Full jet reconstruction [2, 3] may enable the study of jet quenching at the partonic level, without the added complexity of hadronization. However, jet reconstruction in heavy ion events is a challenging task, due to the large population of combinatorial background jets generated by the jet reconstruction algorithm from random recombination of particles not correlated via single hard scattering, and to the distortion of the measured energy of true jets by background fluctuations. Experimental control of these effects may be achieved by pruning the soft component of events prior to jet finding, and requiring jet candidates to contain a hard fragmentation component. However, for quenched jets, these analysis techniques may also bias the jet population and the reported jet energy. For accurate quenching measurements it is necessary to assess such biases and to minimize their effects. In these proceedings we present data-driven methods for minimally-biased inclusive and coincidence jet measurements in heavy ion collisions, at both RHIC and LHC. We base our approach on previous developments [4, 5, 6]. Background fluctuations are "unfolded" using an iterative technique incorporating Bayes's Theorem [7]. We employ a model event generator to evaluate these methods, and give prescriptions to minimize fragmentation bias in real data analysis. This approach is applicable to jet measurements by the STAR experiment at 
RHIC and ALICE at the LHC, which are able to reconstruct a large fraction of all jet fragments via precise charged particle tracking and electro-magnetic calorimetry.

\section{Toy Model and Jet Reconstruction}

Our aim is to clarify the generic effects underlying the largest systematic uncertainties in heavy ion jet measurements. For such a parametric study, it is not necessary to model heavy ion events and experimental response in detail; indeed, the complexity of such an approach may obscure the important generic effects. We therefore employ a simplified Toy Model event generator, that nevertheless captures the main features of heavy ion jet reconstruction seen in data [4, 5, 6]. The Toy Model randomly generates massless particles with uniform distribution within $|\eta|<1$ and $0<\varphi<2 \pi$. The transverse momentum distribution of the soft background is a Boltzmann distribution, with $\left\langle p_{\mathrm{T}}\right\rangle=500 \mathrm{MeV}$ at RHIC and $\left\langle p_{\mathrm{T}}\right\rangle=700 \mathrm{MeV}$ at the LHC. The choice of jet distribution depends on the observable: for inclusive jets we utilize the inclusive spectrum measured in $\mathrm{p}+\mathrm{p}$ collisions at the same $\sqrt{s}$, scaled by $T_{A A}$, while for the coincidence measurement we utilize the semi-inclusive distribution calculated by PYTHIA for $\mathrm{p}+\mathrm{p}$ collisions, without additional scaling. Jets are fragmented using the PYTHIA fragmentation routines, or are not fragmented at all; i.e. a single particle carries the total jet momentum ("SP" fragmentation). The total multiplicity per event corresponds to the average multiplicity of all particles (charged plus neutral) in 0-5\% central Au+Au collisions at RHIC or $0-5 \%$ central $\mathrm{Pb}+\mathrm{Pb}$ collisions at the LHC.

Millions of Toy Model event are generated, and are analyzed with the same algorithms used for real data. Jets are reconstructed using the Anti- $k_{T}$ algorithm [8] with resolution parameter $R=0.4$ and energy recombination scheme, and with median background density estimate $\rho$ as described in [9]. We utilize the FASTJET implementation with default settings [10]. Jet reconstruction incorporates all particles with $p_{T}>0.2 \mathrm{GeV} / \mathrm{c}$ and $|\eta|<1.0$, with full azimuthal coverage. Accepted jets have $|\eta|<0.6$ and area $A_{\text {jet }}>0.4$ [4]. The measured jet energy $p_{T}^{\text {rec }}$ is corrected on an event-wise basis via [9]:

$$
p_{T}^{\langle\text {corr }}=p_{T}^{\text {rec }}-\rho \cdot A_{\text {jet }},
$$

where $\rho$ is the event-wise estimate of the background density (energy per unit area) and (corr〉 indicates event-wise correction for background. Local variations of background density relative to $\rho$ will generate large distortions in the distribution of $p_{T}^{\text {〈corr }}$, which must be corrected via unfolding. We use iterative unfolding incorporating Bayes's Theorem [7], with the response matrix corresponding to $\delta p_{\mathrm{T}}$, the distribution of fluctuations in jet response measured using data [4, 6]. In this case, the "data" are Toy Model events.

The results presented here are for SP fragmentation, in order to isolate the biases in the jet measurement due to suppression of the combinatorial background component and unfolding of background fluctuations. The validity of the SP approach is based on the observed insensitivity of the response of the Anti- $k_{T}$ algorithm in heavy ion events to the detailed pattern of fragmentation of particles measured within the jet cone [4, 5]. Figure 1 shows the generated particle distribution for SP fragmentation, together with the reconstructed $\left(p_{T}^{\langle\text {corr }}\right)$ jet spectrum, for central $\mathrm{Au}+\mathrm{Au}$ collisions at RHIC.
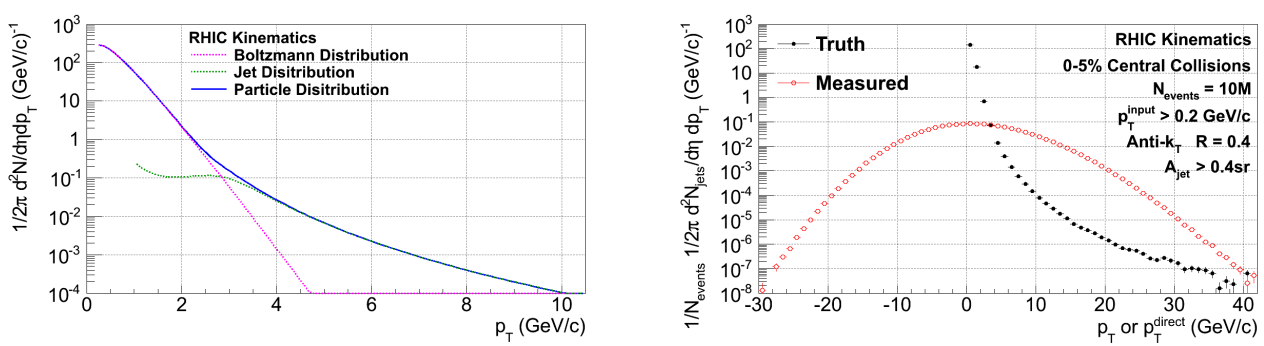

Figure 1. Toy Model distributions for central Au+Au collisions at RHIC. Left: particle distribution with SP fragmentation. Right: particle ("Truth") and reconstructed jet spectra. 

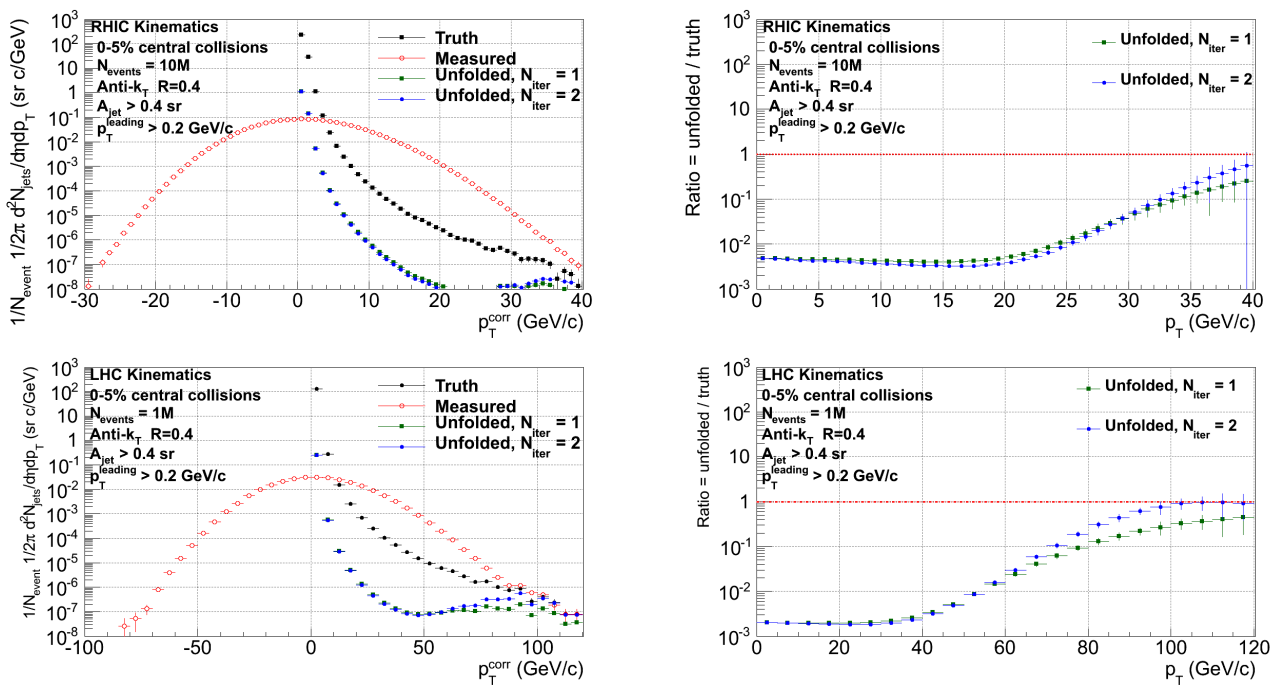

Figure 2. Measured, truth and unfolded distributions for fully inclusive jets. Upper: RHIC; lower: LHC. Right plots show ratio Unfolded/Truth.

\section{Inclusive Jet Spectrum Measurement}

We first consider measurement of the fully inclusive jet spectrum. Figure 2 shows the Truth, measured, and unfolded jet distributions for RHIC (upper panels) and LHC (bottom panels). The distributions resulting from the first two Bayesean unfolding iterations disagree strongly with the Truth distribution, and successive iterations do not reduce the discrepancy. This effect is due to the presence of an overwhelming population of combinatorial background jets which do not have an underlying physical distribution, but which the unfolding algorithm cannot distinguish from true jets. Figure 3 shows the same calculation, but with the distribution to be unfolded restricted to jets containing a leading particle $p_{T}^{\text {leading }}>4.0 \mathrm{GeV} / \mathrm{c}$ at RHIC and $p_{T}^{\text {leading }}>10.0 \mathrm{GeV} / \mathrm{c}$ at LHC. This cut strongly suppresses the combinatorial jet background distribution, and the resulting Unfolded distribution corresponds to the Truth distribution within $10 \%$ for all $p_{\mathrm{T}}$.
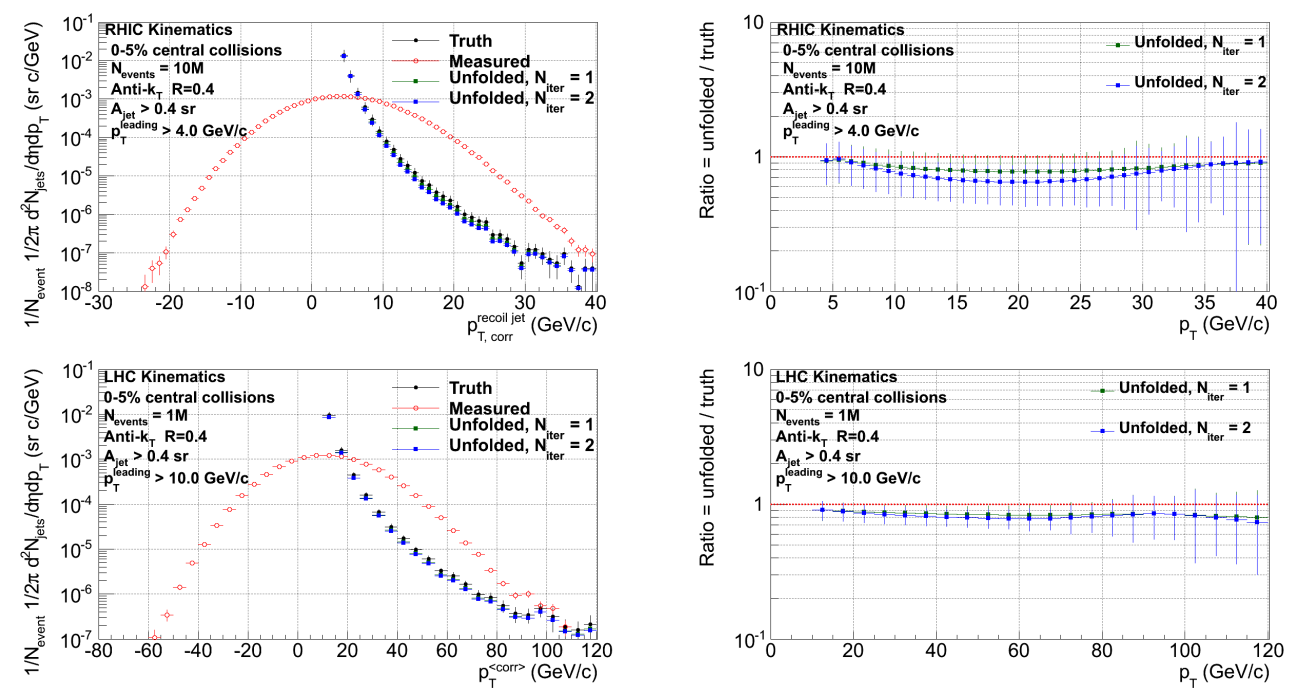

Figure 3. Measured, truth and unfolded distributions for inclusive, jets with leading particle above a given threshold. RHIC (upper plots) and LHC (bottom plots). 
The calculations presented here employ simplified SP fragmentation, though calculations employing PYTHIA fragmentation show similar behavior as a function of the $p_{T}^{\text {leading }}$ threshold. The behavior in data will depend on the detailed interplay of (quenched) jet fragmentation and the background hadron distribution, including effects of flow, which are not included in the Toy Model.

It is apparent that accurate unfolding of background fluctuations from the inclusive spectrum requires suppression of the combinatorial background, via imposition of a fragmentation bias (see also [11]). However, this approach also biases the resulting true jet population, which may be significant for quenching measurements. The lowest threshold value for $p_{T}^{\text {leading }}$ that enables stable unfolding of the background fluctuations is clearly preferred, but quantitative assessment of the remaining biases in such an approach are beyond the scope of this model study.

Given effective suppression of the combinatorial jet population, the precision of the inclusive measurement is then limited by the precision with which the unfolding response matrix is known. The response matrix includes contributions both from detector effects and from the fluctuating event background, with the latter generally dominant in heavy ion measurements. Data-driven techniques [4, 5, 6] are able to measure the fluctuation distribution $\delta p_{\mathrm{T}}$ over several decades in magnitude.

\section{Hadron+Jet Coincidence Measurement}

We next turn to a coincidence measurement, the semi-inclusive rate of jets recoiling against a high $p_{\mathrm{T}}$ hadron trigger (" $\mathrm{h}+$ jet"). The acceptance for the recoil jet is

$$
\left|\varphi_{\text {hadron }}-\varphi_{\text {jet }}-\pi\right|<\pi / 4
$$

The basic observable is the $p_{\mathrm{T}}$ distribution of the number of observed recoil jets, normalized by the number of triggers. We prefer a hadron to a jet trigger, since hadrons can be accurately measured in heavy ion collisions without having to account for complex background effects, and their (suppressed) production is well-understood in jet quenching models. In addition, model studies suggest that a high $p_{\mathrm{T}}$ hadron trigger imposes a significant "surface" bias on the measured population, corresponding to maximum the path length in matter for recoiling jets, whereas a jet trigger does not [12, 13].
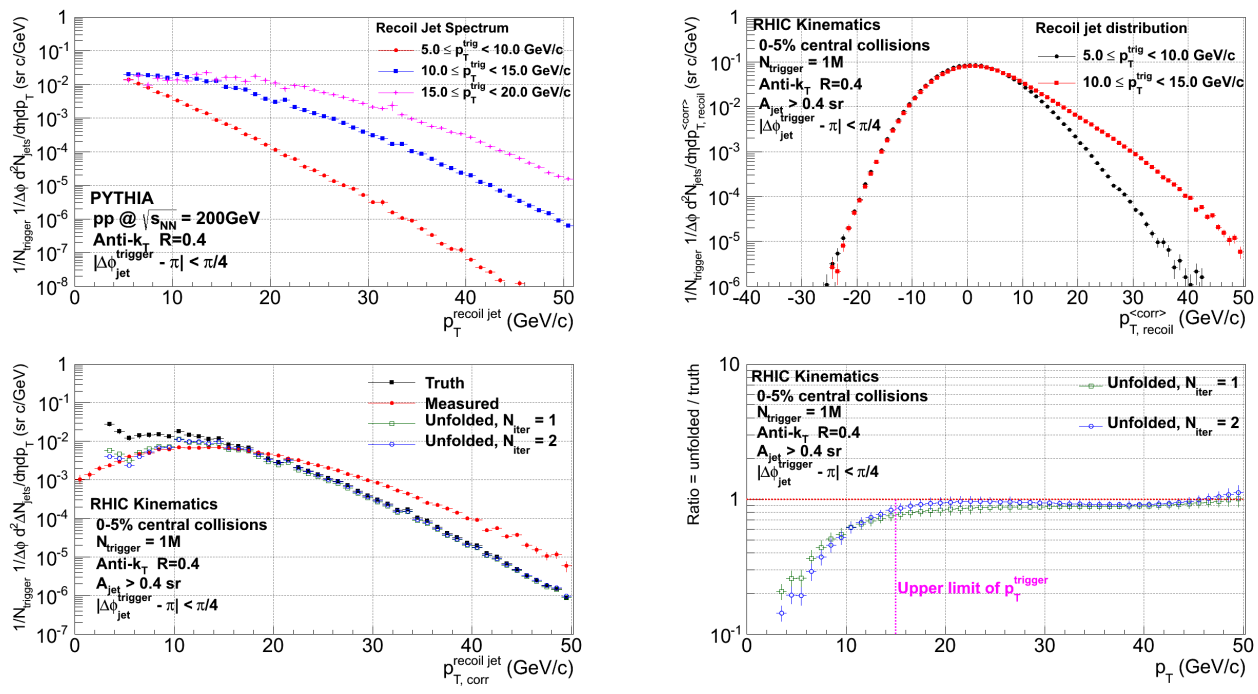

Figure 4. h+jet measurements at RHIC $(\sqrt{s}=200 \mathrm{GeV})$ : semi-inclusive jet distributions. Error bars are from event generation and correspond to very large integrated luminosity. Upper left: PYTHIA calculation for $\mathrm{p}+\mathrm{p}$ collisions with various intervals of trigger hadron $p_{\mathrm{T}}$. Remaining panels are Toy Model calculations for central Au+Au collisions. Upper right: $p_{T}^{\langle\text {corr }}$ distribution for two intervals of trigger hadron $p_{\mathrm{T}}$; lower left: difference distribution and results of unfolding; lower right: ratio Unfolded/Truth.

Fig. 4 upper left panel, shows a PYTHIA calculation of the semi-inclusive $\mathrm{h}+$ jet distribution for $\mathrm{p}+\mathrm{p}$ collisions at RHIC $\sqrt{s}=200 \mathrm{GeV}$, for three different intervals of hadron trigger $p_{\mathrm{T}}$. Note that the recoil jet distribution depends 
strongly on hadron trigger $p_{\mathrm{T}}$, and the probability to observe a recoil jet in the acceptance with $p_{\mathrm{T}}$ above the hadron trigger threshold is at most a few percent. Fig. 4, upper right, shows the equivalent recoil jet distribution for central $\mathrm{Au}+\mathrm{Au}$ collisions, for two intervals of trigger hadron $p_{\mathrm{T}}$. Unfolding of background fluctuations for these individual distributions fails in a way similar to unfolding of fully inclusive distributions (Fig. 2), and for the same reason: the recoil region is dominated by combinatorial background jets, even for a high $p_{\mathrm{T}}$ trigger, due to the low probability for true coindiences. In order to overcome this background effect without imposing a fragmentation bias on the recoil jet population, we note that the combinatorial jet distribution is, by definition, independent of trigger $p_{\mathrm{T}}$. This invariance is seen in the upper right panel, which compares the recoil $p_{T}^{\langle\text {corr }\rangle}$ distribution for two different trigger $p_{\mathrm{T}}$ ranges. The region $p_{T}^{\langle\text {corr }\rangle}<0$, where the combinatorial background component may be expected to dominate, is very similar for the two trigger intervals, whereas the region for large positive $p_{T}^{\langle\text {corr }\rangle}>0$ exhibits strong correlation with trigger $p_{\mathrm{T}}$. This invariance may not be precise in real data analysis, due to biases in reaction plane orientation and centrality imposed by the requirement of a high $p_{\mathrm{T}}$ trigger hadron. Such biases are not present in Toy Model events. Their effects in real data analysis can however be minimized, as described below.

We exploit this invariance by defining a new observable: the difference between the two distributions in Fig. 4. upper right panel, which represents the evolution of the recoil distribution with trigger $p_{\mathrm{T}}$. The hadron trigger $p_{\mathrm{T}}$ for both ranges should be chosen to be high enough that the probability per event for such a trigger is low, so that a hard recoil jet most likely originates from the same hard interaction as the trigger. In addition, for sufficiently high $p_{\mathrm{T}}$ hadron triggers, the reaction plane and centrality biases of the trigger have weak, if any, dependence on trigger $p_{\mathrm{T}}$, and such biases will affect the combinatorial background similarly for the two trigger $p_{\mathrm{T}}$ ranges. After accounting for the strict conservation of jet density [5], the combinatorial background jet component can be suppressed in the difference distribution in a purely data-driven way, at the per-mil level of precision or better. Fig. 4, lower left, shows the difference distribution ("Measured", red points), which represents the hard recoil jet distribution, but with energy still smeared by background fluctuations. The lower left panel also shows the result of unfolding of these fluctuations compared to the Truth distribution from PYTHIA $\mathrm{p}+\mathrm{p}$ events, with the ratio Unfolded/Truth shown in lower right panel. The Truth distribution is recovered within $\sim 10 \%$ precision over a broad kinematic range above the trigger threshold, with minimal cut on the jet constituents $\left(p_{\mathrm{T}}>200 \mathrm{MeV}\right)$ and without the imposition of any fragmentation bias on the measured jet population. Similar results are obtained for $h+j e t$ measurements at the LHC, with appropriate choice of trigger $p_{\mathrm{T}}$.

\section{References}

[1] A. Majumder, M. Van Leeuwen, The Theory and Phenomenology of Perturbative QCD Based Jet Quenching, Prog.Part.Nucl.Phys. A66 (2011) 41-92. arXiv:1002.2206 doi:10.1016/j.ppnp.2010.09.001

[2] G. Aad, et al., Observation of a Centrality-Dependent Dijet Asymmetry in Lead-Lead Collisions at sqrt(S(NN))=2.76 TeV with the ATLAS Detector at the LHC, Phys.Rev.Lett. 105 (2010) 252303. arXiv:1011.6182 doi:10.1103/PhysRevLett.105.252303

[3] S. Chatrchyan, et al., Observation and studies of jet quenching in $\mathrm{PbPb}$ collisions at nucleon-nucleon center-of-mass energy $=2.76 \mathrm{TeV}$, Phys.Rev. C84 (2011) 024906. arXiv: 1102.1957. doi:10.1103/PhysRevC.84.024906

[4] P. M. Jacobs, Background Fluctuations in Heavy Ion Jet Reconstruction, arXiv:1012.2406v2 [nucl-ex].

[5] G. de Barros, Inclusive Distribution of Fully Reconstructed Jets in Heavy Ion Collisions at RHIC: Status Report, AIP Conf.Proc. 1441 (2012) 825-828. arXiv:1109.4386 doi:10.1063/1.3700690

[6] B. Abelev, et al., Measurement of Event Background Fluctuations for Charged Particle Jet Reconstruction in Pb-Pb collisions at $\sqrt{s_{N N}}=2.76$ TeV, JHEP 1203 (2012) 053. arXiv: 1201.2423 doi:10.1007/JHEP03(2012) 053

[7] G. D'Agostini, A Multidimensional unfolding method based on Bayes' theorem, Nucl.Instrum.Meth. A362 (1995) 487-498. doi : 10.1016/ 0168-9002(95) 00274-X

[8] M. Cacciari, G. P. Salam, G. Soyez, The anti- $k_{T}$ jet clustering algorithm, JHEP 04 (2008) 63.

[9] M. Cacciari, G. P. Salam, Pileup subtraction using jet areas, Phys. Lett. B659 (2008) 119-126.

[10] M. Cacciari, G. P. Salam, G. Soyez, FastJet 2.4.4 user manual (2010). URL http://fastjet.fr/

[11] J. Hanks, A. Sickles, B. Cole, A. Franz, M. McCumber, et al., Jet-Underlying Event Separation Method for Heavy Ion Collisions at the Relativistic Heavy Ion Collider. arXiv: 1203.1353

[12] T. Renk, K. J. Eskola, Prospects of medium tomography using back-to-back hadron correlations. Phys. Rev. C 75 (2007) 054910. doi: 10.1103/PhysRevC.75.054910

URL http://link.aps.org/doi/10.1103/PhysRevC.75.054910

[13] T. Renk, Sensitivity of the dijet asymmetry to the physics of jet quenching Phys. Rev. C 85 (2012) 064908. doi:10.1103/PhysRevC.85. 064908

URL http://link.aps.org/doi/10.1103/PhysRevC.85.064908 